\title{
Changing Patterns of Mental Health Care Use: The Role of Integrated Mental Health Services in Veteran Affairs Primary Care
}

\author{
Lucinda B. Leung, MD, PhD, MPH, Jean Yoon, PhD, Lisa V. Rubenstein, MD, MSPH, \\ Edward P. Post, MD, PhD, Maureen E. Metzger, PhD, MPH, \\ Kenneth B. Wells, MD, MPH, Catherine A. Sugar, PhD, and José J. Escarce, MD, PhD
}

Objective: Aiming to foster timely, high-quality mental health care for Veterans, VA's Primary CareMental Health Integration (PC-MHI) embeds mental health specialists in primary care and promotes care management for depression. PC-MHI and patient-centered medical home providers work together to provide the bulk of mental health care for primary care patients with low-to-moderate-complexity mental health conditions. This study examines whether increasing primary care clinic engagement in PC-MHI services is associated with changes in patient health care utilization and costs.

Methods: We performed a retrospective longitudinal cohort study of primary care patients with identified mental health needs in 29 Southern California VA clinics from October 1, 2008 to September 30, 2013, using electronic administrative data $(n=66,638)$. We calculated clinic PC-MHI engagement as the proportion of patients receiving PC-MHI services among all primary care clinic patients in each year. Capitalizing on variation in PC-MHI engagement across clinics, our multivariable regression models predicted annual patient use of 1) non-primary care based mental health specialty (MHS) visits, 2) total mental health visits (ie, the sum of MHS and PC-MHI visits), and 3) health care utilization and costs. We controlled for year- and clinic-fixed effects, other clinic interventions, and patient characteristics.

Results: Median clinic PC-MHI engagement increased by 8.2 percentage points over 5 years. At any given year, patients treated at a clinic with 1 percentage-point higher PC-MHI engagement was associated with $0.5 \%$ more total mental health visits (CI, $0.18 \%$ to $0.90 \% ; P=.003$ ) and $1.0 \%$ fewer MHS visits $(\mathrm{CI},-1.6 \%$ to $-0.3 \% ; P=.002)$; this is a substitution rate, at the mean, of $1.5 \mathrm{PC}-\mathrm{MHI}$ visits for each MHS visit. There was no PC-MHI effect on other health care utilization and costs.

Conclusions: As intended, greater clinic engagement in PC-MHI services seems to increase realized accessibility to mental health care for primary care patients, substituting PC-MHI for MHS visits, without increasing acute care use or total costs. Thus, PC-MHI services within primary care clinics may improve mental health care value at the patient population level. More research is needed to understand the relationship between clinic PC-MHI engagement and clinical quality of mental health care. (J Am Board Fam Med 2018;31:38-48.)

Keywords: Health Care Costs, Depressive Disorder, Mental Health, Patient-centered Care, Primary Health Care, Retrospective Studies, Veterans

Managed and accountable care organizations, like the US Department of Veterans Affairs (VA), strive to provide access to timely, equitable mental and

This article was externally peer reviewed.

Submitted 11 April 2017; revised 10 August 2017; accepted 14 August 2017.

From the VA Health Services Research and Development (HSR\&D) Center for the Study of Healthcare Innovation, Implementation, \& Policy, Greater Los Angeles VA Healthcare System, Los Angeles, CA (LBL, LVR, KBW); Division physical health care for enrollees. ${ }^{1}$ Veterans who use VA care have been found to have a high burden of mental illness; approximately $30 \%$ of patients visiting VA primary care have a diagnosed mental

of General Internal Medicine and Health Services Research, University of California-Los Angeles (UCLA) David Geffen School of Medicine, Los Angeles (LBL, LVR, JJE); VA Health Economics Resource Center, VA Palo Alto Healthcare System, Menlo Park, CA (JY); Department of General 
health disorder. ${ }^{2}$ Mental health services that are physically and organizationally separate from primary care may not be able to meet the needs of this large population. As a result, the VA is uniquely positioned to leverage team-based care models that integrate physical and mental health care.

To improve mental health care quality and access among Veterans, the VA mandated Primary Care-Mental Health Integration (PC-MHI) in all large primary care clinics nationwide beginning in 2007. ${ }^{3}$ The initiative provided programmatic technical assistance, education and training, and data sources for quality improvement ${ }^{4}$ to facilitate the implementation of a "blended model that includes colocated collaborative care and care management." ${ }^{5}$ PC-MHI embedded mental health specialists (eg, psychologists, social workers, licensed mental health counselors) in primary care clinics, and promoted nurse care management ${ }^{6}$ informed by evidence-based models such as collaborative care. ${ }^{7}$ In contrast with traditional mental health services, PC-MHI services are delivered in primary care, are brief and limited in number, are delivered by midlevel providers in consultation with psychiatrists, and targeted at depression, anxiety, and alcohol misuse. $^{8}$

VA investment in PC-MHI is notable because evidence-based integrated care models are often difficult to disseminate and implement in realworld health care delivery systems. ${ }^{9}$ In addition to monitoring PC-MHI service volume using admin-

Internal Medicine, University of California-San Francisco School of Medicine, San Francisco, CA (JY); RAND Corporation, Santa Monica, CA (LVR, KBW); UCLA School of Public Health, Los Angeles (LVR, KBW, CAS, JJE); VA Center for Clinical Management Research, Ann Arbor, MI (EPP, MEM); University of Michigan Medical School, Ann Arbor (EPP); UCLA Center for Health Services and Society, Los Angeles (KBW); ${ }^{10}$ UCLA Semel Institute for Neuroscience and Human Behavior, Los Angeles (KBW, CAS).

Funding: Support for this article was provided by the Robert Wood Johnson Foundation VA Clinical Scholars ${ }^{\circledR}$, VA Quality Scholars, and UCLA Specialty Training and Advanced Research programs. The views expressed in this article are those of the authors and do not necessarily reflect the position or policy of the Department of Veterans Affairs or the US government.

Conflict of interest: none declared.

Prior presentations: We presented earlier versions of the manuscript at the 2016 Society of General Internal Medicine National Meeting in Hollywood, FL; 2016 AcademyHealth Annual Research Meeting in Boston, MA; and 2017 VA HSR\&D/QUERI National Conference in Arlington, VA.

Corresponding author: Lucinda B. Leung, MD, PhD, MPH, 11301 Wilshire Blvd (111G), Building 500, Room 3244, Los Angeles, CA 90073 (E-mail: lleung@mednet.ucla.edu). istrative data, VA's National PC-MHI Evaluation regularly surveys program implementation efforts and has demonstrated appropriate mental health staffing in primary care and a service focus on depression, anxiety, post-traumatic stress disorder (PTSD), and alcohol misuse. ${ }^{10}$ Having a PC-MHI program in a primary care clinic has been associated with increased diagnosis of mental health disorders. ${ }^{2}$ Patients who had not recently used VA services have been found more likely to use PCMHI services than others. ${ }^{11}$ Individual contact with PC-MHI programs has also been associated with improved outcomes, such as increased completion of mental health specialty (MHS) referral ${ }^{12}$, increased odds of PTSD diagnosis and treatment initiation $^{13}$, and lower risk of having an emergency department (ED) visit, hospitalization, or death. ${ }^{14}$

Supported by additional primary care staffing through the VA patient-centered medical home initiative (ie, Patient Aligned Care Teams [PACT]) in $2010^{15}$, the aim has been to provide the bulk of mental health care for primary care patients with low-to-moderate-complexity mental health conditions within the medical home. ${ }^{8}$ Recently, researchers have observed reductions in MHS visits and total VA costs and have attributed this to PACT. ${ }^{16,17}$ However, it remains unclear what role PC-MHI services may have played in accounting for these reductions. Studies that attempted to isolate the effect of PC-MHI on mental health care utilization have yielded mixed results. ${ }^{10,18}$ To date, no study has longitudinally examined clinic PC$\mathrm{MHI}$ engagement (ie, the intensity of the clinic population's PC-MHI service use) to understand PC-MHI's impact on health care utilization and costs.

We used 5 years of electronic administrative patient data to evaluate the relationship between clinic PC-MHI engagement and mental health care utilization in a large regional cohort of VA patients. We hypothesized that greater clinic PC-MHI engagement (ie, through greater clinic uptake of PCMHI services) would be associated with more VAprovided mental health services overall and less non-primary care-based MHS services. We secondarily hypothesized that greater clinic PC-MHI engagement would be associated with decreased total cost of VA care through potential effects on the full range of health care utilization, particularly among patients with high levels of comorbidity, whom are often high health care utilizers. 


\section{Methods}

\section{Study Design, Setting, and Participants}

We performed a retrospective longitudinal patient cohort study from fiscal years (FYs) 2009 to 2013 (October 1, 2008 to September 30, 2013). This study used data originally obtained to examine the impact of patient-centered medical home implementation using evidence-based quality improvement. Eligible patients were VA primary care users having at least 2 primary care visits in the baseline year. To understand mental health care utilization in those with the greatest need, we chose to examine patients diagnosed with 1 or more of the following conditions on at least 2 separate encounters during the study period: alcohol use disorders, substance use disorders, depression, bipolar disorder, schizophrenia, PTSD, personality disorders, anxiety and other disorders $(n=66,638)$.

Study patients visited 1 of 29 primary care clinics (4 hospital based, 25 community based) in Southern California. Although most clinics had fewer than 5000 patients per year and were not mandated to implement $\mathrm{PC}-\mathrm{MHI}$ programs, 27 of 29 clinics recorded $\mathrm{PC}-\mathrm{MHI}$ encounters during the study period, indicating robust uptake of PC-MHI services in this region. Six clinics concurrently participated in an evidence-based quality improvement intervention to facilitate PACT adoption (EBQIPACT). ${ }^{19}$

\section{Measures}

\section{Data Source}

We obtained the number of outpatient encounters, hospitalizations, and costs of direct VA-provided care in each study year for each study patient from the VA's National Patient Care Databases and the Decision Support System files.

\section{Clinic Assignment}

We assigned each patient to a home primary care clinic site by determining where the patient received a plurality of primary care visits during the baseline year (FY 2009). If there was a tie between 2 or more clinics, we preferentially assigned patients to community-based clinics and then, if still tied, to the most recent clinic visited for primary care.

\section{Main Outcomes}

Our main outcomes were 1) MHS visits, defined as the number of visits to a non-primary care-based mental health provider for each patient (ie, excludes PC-MHI visits), and 2) total mental health visits, defined as the sum of each patient's MHS and PC-MHI visits. Given the skewed distribution, we dropped extreme values of all mental health visits (ie, greater than 3 standard deviations $[\sim 90$ visits]) in each study year.

\section{Secondary Outcomes}

We grouped and counted all clinical visit codes related to primary care (excluding $\mathrm{PC}-\mathrm{MHI})$, nonmental health specialties, ED visits, and hospitalizations across all diagnoses and departments. We excluded FY 2009 ED visits due to inconsistent reporting and further subdivided ED visits (eg, ambulatory care sensitive condition related, mental health/substance abuse related). We did not examine telephone, laboratory, radiology, and administrative visits. We aggregated health care costs from all VA-sponsored care (ie, care paid for by the VA) for each patient in each year and adjusted costs to 2013 dollars using the general consumer price index.

\section{Main Predictor}

Our main predictor was clinic PC-MHI engagement, defined as the number of PC-MHI service users divided by the number of primary care patients in each clinic during each year. PC-MHI service users are those who visited PC-MHI at least once during a given study year, ascertained from nationally designated electronic PC-MHI encounter codes (534 and 539) For ease of interpretation in descriptive analyses, we dichotomized clinics by whether they fell above ("high PC-MHI engagement") or below ("low PC-MHI engagement") the baseline median. Because we hypothesized that there might be a linear relationship, we used clinic PC-MHI engagement as a continuous variable in all regression analyses.

\section{Covariates}

Our study controlled for patient characteristics affecting health care utilization, including age, sex, race-ethnicity, marital status, non-VA health insurance, VA care eligibility (ie, qualification for VA health care benefits based on duty requirements, discharge conditions, etc.), level of service-connected disability (ie, degree to which a given injury or condition can be attributed to military service experiences), homelessness, and distance from 
home address to primary care clinic. We adjusted for mental health diagnoses based on International Classification of Diseases, Ninth Revision encounter codes and calculated the Charlson Comorbidity Index using the Deyo-Quan approach for each patient's physical comorbidities in each year. ${ }^{20,21}$ To control for time-varying clinic characteristics, we identified the 6 EBQI-PACT sites through an indicator for EBQI-PACT participation and implementation year. In addition, we examined the following time-invariant clinic characteristics: type (ie, hospital based vs community based), location, and size.

\section{Analysis}

In descriptive analyses, we examined clinic PCMHI engagement for each study year. We analyzed mean numbers of medical visits and health care costs per patient as well as the percent they changed between the baseline to final study year. Furthermore, we compared patient- and clinic-level characteristics of high and low PC-MHI engagement clinics using $t$ - and $\chi^{2}$ tests at baseline. Using unadjusted regression models with indicator variables for each year, we estimated the association of clinic PC-MHI engagement on our health care utilization and cost outcomes.

We used multivariable analyses to estimate the effects of clinic PC-MHI engagement on our dependent variables, controlling for utilization-related patient characteristics, clinic EBQI-PACT participation, and year and clinic fixed effects. All regression models included year and clinic fixed effects to control for any secular trends and invariant clinic characteristics. Models included patient random effects to account for the multiple nonindependent observations per patient over the 5 study years. We adjusted standard errors for clustering of patients within clinics. To account for overdispersion in the distributions of our health care utilization counts, we used multi-level negative binomial regression models and derived incidence rate ratios. To account for the skewed distribution of health care costs in each year, we used logtransformed costs in our multi-level linear regression models.

In sensitivity analyses, we 1) stratified patients by whether their clinics were required to have PCMHI programs, 2) included patients without mental health diagnoses $(n=112,737), 3)$ separately analyzed patients with multiple chronic comorbidi- ties (ie, Charlson Comorbidity Index of 2 or higher) ( $\mathrm{n}=18,362), 4)$ excluded patients who were age 65 years or older and eligible for Medicare coverage ( $\mathrm{n}=21,510)$, and 5) excluded patients who had no visits during the final study year (ie, left VA care) or died during the study period ( $\mathrm{n}=$ 10,203). We additionally examined whether there were any mortality differences (ie, number of patient deaths) associated with increasing clinic PC$\mathrm{MHI}$ engagement. We determined significance using a 2-tailed $\alpha$ of 0.05 and conducted all analyses in Stata 14.0, College Station, TX. The Greater Los Angeles VA Institutional Review Board approved this study.

\section{Results}

\section{Unadjusted analyses}

Over 5 years, median clinic PC-MHI engagement across study clinics increased by $8.2 \%$ (Figure 1). We found significant baseline differences between patients in high and low PC-MHI engagement clinics (grouped per FY 2009 clinic PC-MHI engagement median, $1.1 \%$; range, $0 \%$ to $15.9 \%$ ) (Tables 1 and 2). Compared with low PC-MHI engagement clinics, high $\mathrm{PC}-\mathrm{MHI}$ engagement clinics had greater patient volume, were more likely to be hospital based, and included the 1 nonmetropolitan clinic and all clinics participating in EBQI-PACT. Compared with patients in low PC$\mathrm{MHI}$ engagement clinics, those in high $\mathrm{PC}-\mathrm{MHI}$ engagement clinics were more likely to be older, chronically ill, male, Black, single, uninsured (ie, without non-VA insurance), homeless, to live farther from their home primary care clinic, to have lower eligibility ranking for VA care, and to less often have service-connected disabilities. High and low PC-MHI engagement clinics had similar proportions of patients with bipolar disorder and schizophrenia.

From the baseline to final study year, patients seemed to have fewer total mental health visits $(-8.0 \%)$ and MHS visits $(-15.0 \%)$. Patients in high PC-MHI engagement clinics used mental health services more frequently and experienced smaller reductions over time, compared with those in low PC-MHI engagement clinics. In unadjusted analyses, we found that, at any given year, patients treated at a clinic with a 1 percentage-point higher PC-MHI engagement rate had significantly less MHS, total mental health, primary care, and ED 
Figure 1. Distribution of annual Primary Care-Mental Health Integration (PC-MHI) engagement for each clinic. Each point represents a PC-MHI engagement rate for 1 clinic in a given year. Dashed trend lines represent 25th and 75th quartiles. Solid trend line represents the median.

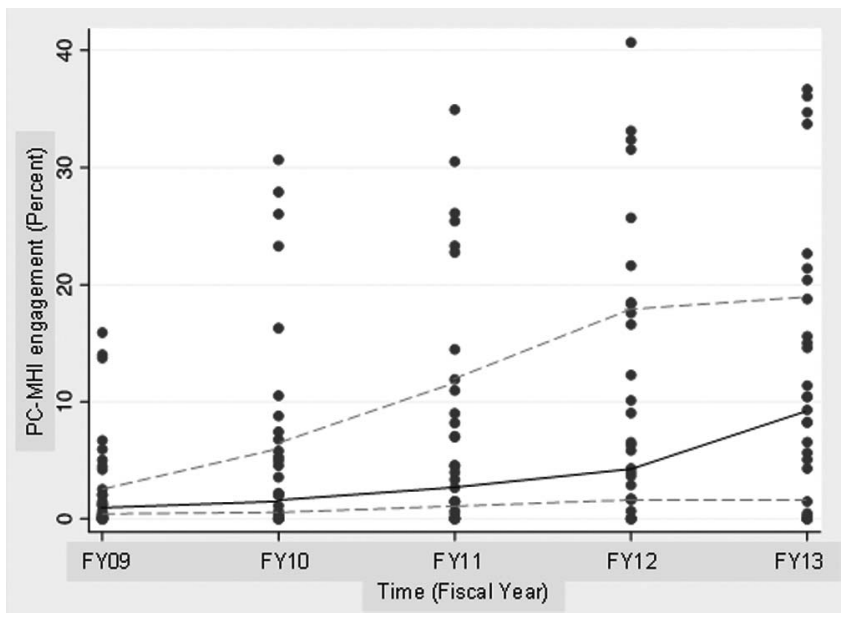

visits and total VA health care costs; however, differences in other specialty visits and hospitalizations were not significant (Appendix 1).

\section{Adjusted Analyses}

After adjusting for patient- and clinic-level factors, however, we found that higher clinic PC-MHI engagement was associated with lower MHS and higher total mental health visit rates. At any given year, patients treated at a clinic with a 1 percentage-point higher PC-MHI engagement rate was associated with a $1.0 \%$ lower MHS visit rate (CI, $-1.6 \%$ to $-0.3 \% ; P=.002)$ and a $0.5 \%$ higher total mental health visit rate (CI, $0.18 \%$ to $0.90 \%$; $P=.003)$. As such, we observed a substitution rate, at the mean, of 1.5 PC-MHI visits for each MHS visit. There was no evidence of a clinic PC-MHI engagement effect on other health care utilization outcomes, including primary care visits, other specialty visits, ED visits, or hospitalizations. Finally, we observed a small but nonsignificant reduction in total costs per year associated with clinic PC-MHI engagement (Figure 2 and Appendix 1).

We observed progressive reductions in total mental health usage over time and lower usage in clinics with PACT implementation support (ie, EBQI-PACT) than those without. Older age and male sex were associated with lower mental health care utilization; however, being single/uninsured/ homeless, living closer to clinic, and having a service-connected disability/multiple chronic comorbidities/any mental health diagnosis except sociopathy were associated with higher utilization. There were no significant racial-ethnic differences in mental health care utilization (Appendix 1).

\section{Sensitivity analyses}

When we stratified analyses by whether patients belonged to a clinic mandated or not to have PC$\mathrm{MHI}$, there was no evidence of clinic PC-MHI engagement effect on MHS visits or total mental health visits in clinics where PC-MHI was required. We, however, found a significant association between clinic PC-MHI engagement and both MHS visits (difference, $-1.3 \%$; CI, $-2.2 \%$ to $-0.3 \% ; P=.01)$ and total mental health visits (difference, $0.7 \%$; CI, $-0.4 \%$ to $1.1 \% ; P<.001$ ) in clinics where it was not required (ie, less than 5000 patients per year), but this difference was not significant when we included an interactive effect with clinic PC-MHI engagement.

Sensitivity analyses on all patients including those without mental health diagnoses, patients with multiple chronic comorbidities, patients younger than 65 years (ie, not Medicare eligible), and patients who left VA care or died before the end of the study, yielded similar results to those reported above. Finally, we found no evidence of a PC-MHI effect on patient mortality.

\section{Discussion}

VA primary care clinics that were more highly engaged in PC-MHI appeared to have higher total 
Table 1. Patient Characteristics by Clinic PC-MHI Engagement in Baseline Year

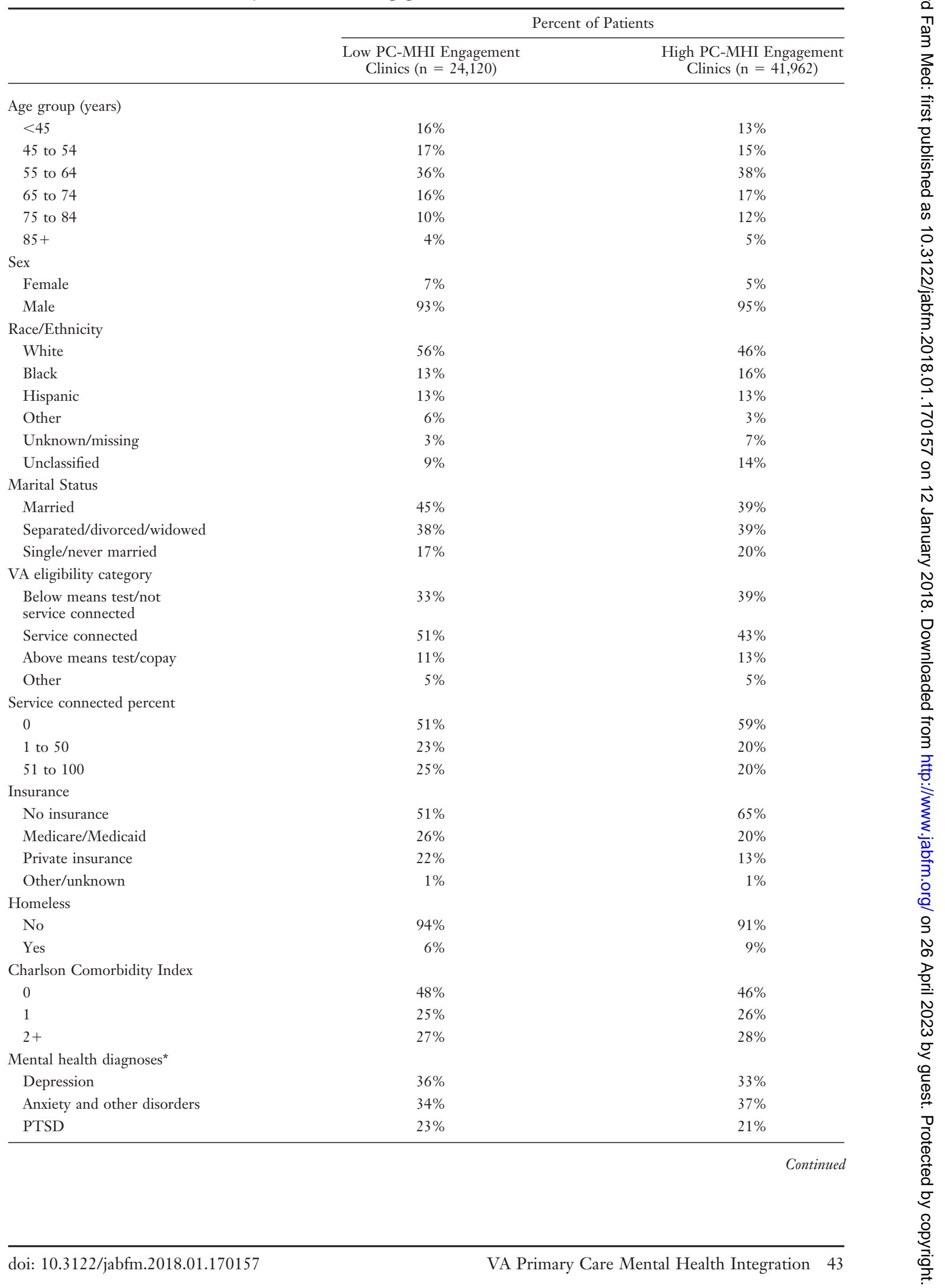




\begin{tabular}{|c|c|c|}
\hline & \multicolumn{2}{|c|}{ Percent of Patients } \\
\hline & $\begin{array}{l}\text { Low PC-MHI Engagement } \\
\quad \text { Clinics }(\mathrm{n}=24,120)\end{array}$ & $\begin{array}{l}\text { High PC-MHI Engagement } \\
\text { Clinics }(\mathrm{n}=41,962)\end{array}$ \\
\hline Alcohol use disorder & $13 \%$ & $11 \%$ \\
\hline Substance use disorder & $7 \%$ & $9 \%$ \\
\hline Bipolar disorder $^{\dagger}$ & $5 \%$ & $5 \%$ \\
\hline \multirow[t]{2}{*}{ Schizophrenia $^{\dagger}$} & $4 \%$ & $4 \%$ \\
\hline & \multicolumn{2}{|c|}{ Mean (SD) } \\
\hline Distance from home to clinic & $12.5(12.2)$ & $13.4(13.2)$ \\
\hline
\end{tabular}

All results had $\chi^{2}$ or $t$-test $P<.05$, unless otherwise indicated $(\mathrm{n}=66,078)$.

*Total sum exceeds $100 \%$ as patients may have multiple mental health diagnoses.

${ }^{\dagger} P$-value was not significant at $95 \%$ level.

PC-MHI, Primary Care-Mental Health Integration; PTSD, Post-traumatic Stress Disorder; VA, Veterans Affairs.

mental health utilization, with PC-MHI substituting for MHS visits. Addressing the need to integrate mental health resources into patient-centered medical homes ${ }^{22,23}$, PC-MHI seems to facilitate access to mental health services by primary care

Table 2. Clinic Characteristics by Clinic PC-MHI Engagement in Baseline Year

\begin{tabular}{|c|c|c|}
\hline \multirow[b]{2}{*}{$\begin{array}{l}\text { Clinic-Level } \\
\text { Characteristics }\end{array}$} & \multicolumn{2}{|c|}{ Percent of Clinics } \\
\hline & $\begin{array}{c}\text { Low PC-MHI } \\
\text { Engagement } \\
(\mathrm{n}=15)\end{array}$ & $\begin{array}{c}\text { High PC-MHI } \\
\text { Engagement } \\
(\mathrm{n}=14)\end{array}$ \\
\hline \multicolumn{3}{|l|}{ EBQI-PACT* } \\
\hline Off & $100 \%$ & $64 \%$ \\
\hline On & $0 \%$ & $36 \%$ \\
\hline \multicolumn{3}{|l|}{ Clinic type } \\
\hline VA hospital based & $7 \%$ & $21 \%$ \\
\hline Community based & $93 \%$ & $79 \%$ \\
\hline \multicolumn{3}{|l|}{ Clinic rurality } \\
\hline Metropolitan & $100 \%$ & $93 \%$ \\
\hline Nonmetropolitan & $0 \%$ & $7 \%$ \\
\hline \multicolumn{3}{|l|}{ Clinic size } \\
\hline Less than 5000 patients & $87 \%$ & $64 \%$ \\
\hline 5000 to 9999 patients & $13 \%$ & $14 \%$ \\
\hline \multirow[t]{2}{*}{10,000 or more patients } & $0 \%$ & $21 \%$ \\
\hline & \multicolumn{2}{|c|}{ Mean (SD) } \\
\hline $\begin{array}{l}\text { Distance from clinic to VA } \\
\text { medical center }\end{array}$ & $44.5(52.2)$ & $36.9(41.7)$ \\
\hline
\end{tabular}

All results had $\chi^{2}$ or $t$-test $P<.05$, unless otherwise indicated $(\mathrm{n}=29)$.

*Based on Patient Aligned Care Team having implemented EBQI-PACT by the end of the study period.

${ }^{\dagger} \mathrm{P}$-value was not significant at $95 \%$ level.

EBQI-PACT, evidence-based quality improvement intervention to facilitate PACT adoption; PC-MHI, Primary CareMental Health Integration; SD, Standard Devation; VA, Veterans Affairs. patients with mental health needs. Interestingly, there was a suggestion that this effect may even be driven by smaller clinics, where PC-MHI is not mandated but still desired. Observed study changes were specific to mental health care utilization and not seen in other outpatient care (ie, other specialty, primary care). This is one of the first studies to examine clinic engagement in PC-MHI programs through the intensity of service use within a large VA primary care clinic population. Earlier studies on PC-MHI largely predated the introduction of patient-centered medical homes, characterized the effect of PC-MHI differently, and had mixed conclusions on the relationship between PCMHI and mental health care utilization. ${ }^{18,10}$ These studies were also not designed to assess access to mental health care for the primary care population. Further investigation into PC-MHI's effect on subcategories of MHS care and patient subgroups is needed to illuminate the mechanism behind PCMHI substitution. If this substitution reflects proper assessment and triage of mental health needs of primary care patients, PC-MHI may be a viable solution for the limited capacity of nonprimary care based MHS services.

Importantly, we did not observe any worse health outcomes, either in mortality, increased acute care use (ie, ED visits, hospitalizations), or medical spending for patients in clinics with greater clinic PC-MHI engagement. Lack of change in acute care use contrasts a previous study that found an association between increased contact with care management and increased ED visits. ${ }^{24}$ Furthermore, our study found that higher clinic PC-MHI engagement had a small, nonsignificant reduction 
Figure 2. Adjusted effect of clinic Primary Care-Mental Health Integration (PC-MHI) engagement on health care utilization and costs. We report incidence rate ratios (and confidence intervals) from multilevel negative binomial regression models predicting health care utilization. Models contained fixed effects for year and clinic and random effects for patient. We adjusted each model for PACT implementation support (evidence-based quality improvement intervention to facilitate PACT adoption [EBQI-PACT] status), and patient characteristics (age, sex, race/ethnicity, marital status, Veterans Affairs (VA) eligibility, disability service connection, health insurance, homelessness, distance from home to primary care clinic, Charlson Comorbidity Index, mental health diagnoses) for the 5-year study period. ED, emergency department.

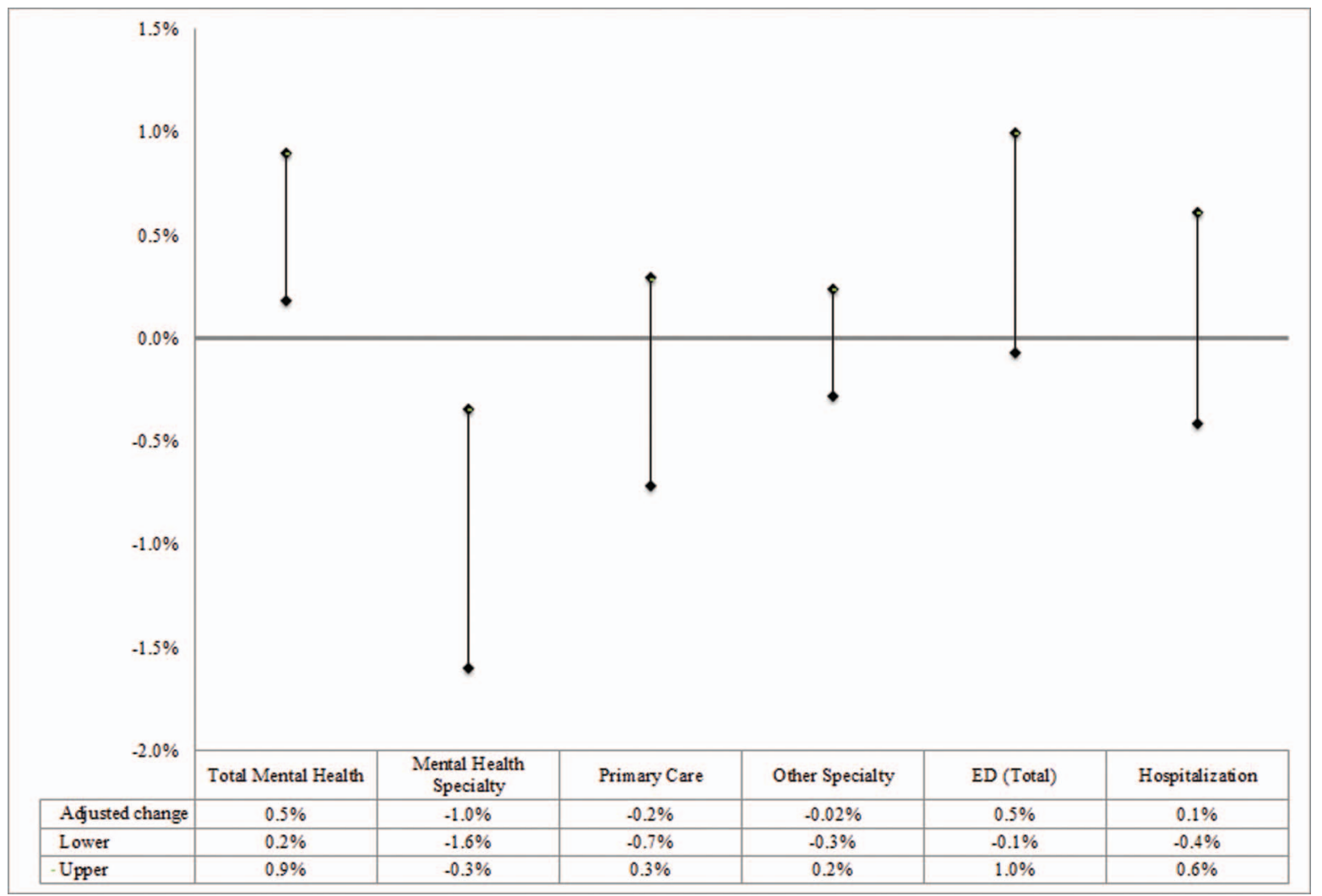

in total cost of care, in some contrast with other studies demonstrating modest additional costs but overall cost effectiveness of collaborative care in other settings. ${ }^{25,26}$ To fully understand the financial burden of these services and whether they can be deemed as high value care, however, requires a more comprehensive cost-analysis that includes program implementation and operation costs.

Our study contributes to the research literature in several ways. To our knowledge, it is the first longitudinal study to examine the full range of health care utilization and medical spending related to $\mathrm{PC}-\mathrm{MHI}$ in the VA, particularly in a region where the uptake of these services is relatively robust. Thus, it is an important addition to literature on large-scale dissemination and implementation of collaborative care, which remains far from usual practice despite strong evidence of effectiveness. ${ }^{9}$ Furthermore, our study uniquely characterizes the difference among clinics by intensity of PC-MHI program uptake, in contrast with previous studies that characterize the presence or absence of a PCMHI program. Thus, in providing additional data beyond that of a dichotomous variable, it may contribute to the development of meaningful and valid quality measures for clinic PC-MHI engagement to assist with the dissemination and implementation of integrated care. ${ }^{27}$

There are several limitations to our research study. First, our longitudinal cohort study design allows use to understand trends in utilization and costs, but it can be limited by patient dropouts, clinic changes, and the exclusion of additional patient enrollees external to the original cohort. Sec- 
ond, we are limited by data incompleteness or inaccuracies, commonly seen with administrative data sources. Two examples were our inability to capture primary care physician provided mental health care and the inconsistent coding of mental health telephone visits. Third, our study examines only health care utilization and medical spending in Southern California's VA health care systems, but does not investigate other measures of clinical quality of care (eg, depression treatment), health outcomes (eg, diabetes complications), social functioning (eg, employment status), or outcomes in other VA clinics. More research on the quality of clinical care, patient outcomes, and the replication of analyses nationally is needed to more accurately assess the health care value of PC-MHI.

Patients with coexisting mental and physical illnesses can be high utilizers of medical care, but may be successfully managed through integrated mental health services. We observed that greater clinic PC-MHI engagement was associated with significantly more total mental health and less MHS visits, likely because patients receive more mental health care through PC-MHI instead of non-primary care based MHS services. Despite the reduction in use of MHS services, greater clinic PCMHI engagement did not seem to have an adverse impact on ED visits, hospitalizations, total patient costs, or mortality. As a result, PC-MHI may improve mental health care value for primary care patients, given that it seems to improve realized accessibility to mental health care without necessarily increasing costs.

The authors would like to acknowledge the Veterans Assessment \& Improvement Laboratory (VAIL) team, Elizabeth $M$. Yano, PhD, MSPH; Jacqueline Fickel, PhD; and Adam Chow for their support of this research.

To see this article online, please go to: bttp://jabfm.org/content/ 31/1/38.full.

\section{References}

1. New Freedom Commission on Mental Health. Achieving the promise: Transforming mental health care in America. Final report. Rockville, MD: U.S. Department of Health and Human Services; 2003.

2. Zivin K, Pfeiffer PN, Szymanski BR, et al. Initiation of primary care-mental health integration programs in the VA Health System: Associations with psychiatric diagnoses in primary care. Med Care 2010;48:843-51.

3. Post EP, Metzger M, Dumas P, Lehmann L. Integrating mental health into primary care within the
Veterans Health Administration. Fam Syst Health 2010;28:83-90.

4. Chang ET, Rose DE, Yano EM, et al. Determinants of readiness for primary care-mental health integration (PC-MHI) in the VA Health Care System. J Gen Intern Med 2013;28:353-62.

5. Department of Veterans Affairs. In: Veterans Health Administration, ed. Uniform mental health services in VA medical centers and clinics. Washington, DC; 2015, 34-6.

6. Pomerantz AS, Sayers SL. Primary care-mental health integration in healthcare in the Department of Veterans Affairs. Fam Syst Health 2010;28:78-82.

7. Archer J, Bower P, Gilbody S, et al. Collaborative care for depression and anxiety problems. Cochrane Database Syst Rev. 2012;10:CD006525.

8. Chang ET, Simon A. Report on Integrating Mental Health Into PACT (IMHIP) in the VA. VA Office of Patient Care Services; 2013.

9. Katzelnick DJ, Williams MD. Large-scale dissemination of collaborative care and implications for psychiatry. Psychiatr Serv 2015;66:904-6.

10. Wray LO, Szymanski BR, Kearney LK, McCarthy JF. Implementation of primary care-mental health integration services in the Veterans Health Administration: Program activity and associations with engagement in specialty mental health services. J Clin Psychol Med Settings 2012;19:105-16.

11. Johnson-Lawrence V, Zivin K, Szymanski BR, Pfeiffer PN, McCarthy JF. VA primary care-mental health integration: Patient characteristics and receipt of mental health services, 2008-2010. Psychiatr Serv 2012;63:1137-41.

12. Davis MJ, Moore KM, Meyers K, Mathews J, Zerth EO. Engagement in mental health treatment following primary care mental health integration contact. Psychol Serv 2016;13:333-40.

13. Bohnert KM, Sripada RK, Mach J, McCarthy JF. Same-day integrated mental health care and ptsd diagnosis and treatment among VHA primary care patients with positive PTSD Screens. Psychiatr Serv 2016;67:94-100.

14. Trivedi RB, Post EP, Sun H, et al. Prevalence, comorbidity, and prognosis of mental health among US veterans. Am J Public Health 2015;105:2564-9.

15. Rosland AM, Nelson K, Sun H, et al. The patientcentered medical home in the Veterans Health Administration. Am J Manag Care 2013;19(7):e263-72.

16. Hebert PL, Liu CF, Wong ES, et al. Patient-centered medical home initiative produced modest economic results for Veterans Health Administration, 2010-12. Health Aff (Millwood) 2014;33:980-7.

17. Yoon J, Chow A, Rubenstein LV. Impact of medical home implementation through evidence-based quality improvement on utilization and costs. Med Care 2016;54:118-25.

18. Pfeiffer PN, Szymanski BR, Zivin K, Post EP, Valenstein M, McCarthy JF. Are primary care mental 
health services associated with differences in specialty mental health clinic use? Psychiatr Serv 2011; 62:422-5.

19. Rubenstein LV, Stockdale SE, Sapir N, et al. A patient-centered primary care practice approach using evidence-based quality improvement: Rationale, methods, and early assessment of implementation. J Gen Intern Med 2014;29(Suppl 2):S589-97.

20. Charlson ME, Pompei P, Ales KL, MacKenzie CR. A new method of classifying prognostic comorbidity in longitudinal studies: Development and validation. J Chronic Dis 1987;40:373-83.

21. Quan H, Sundararajan V, Halfon P, et al. Coding algorithms for defining comorbidities in ICD-9-CM and ICD-10 administrative data. Med Care 2005;43: 1130-9.

22. Croghan TW, Brown JD. Integrating Mental Health Treatment Into the Patient Centered Medical Home. Rockville, MD: Agency for Healthcare Research and Quality; 2010.
23. Baird M, Blount A, Bungardt S, et al. Joint principles: Integrating behavioral health care into the patient-centered medical home. Ann Fam Med 2014; 12:183-5.

24. Waxmonsky J, Verchinina L, Kim HM, et al. Correlates of emergency department use by individuals with bipolar disorder enrolled in a collaborative care implementation study. Psychiatr Serv 2016; appips201500347.

25. Reiss-Brennan B, Briot PC, Savitz LA, Cannon W, Staheli R. Cost and quality impact of Intermountain's mental health integration program. J Healthc Manag 2010;55:97-113; discussion 113-114.

26. Grypma L, Haverkamp R, Little S, Unützer J. Taking an evidence-based model of depression care from research to practice: Making lemonade out of depression. Gen Hosp Psychiatry 2006;28:101-7.

27. Goldman ML, Spaeth-Rublee B, Pincus HA. Quality indicators for physical and behavioral health care integration. JAMA 2015;314:769-70. 


\section{Appendix: Effect of clinic Primary Care-Mental Health Integration (PC-MHI) engagement on healthcare utilization and costs}

\begin{tabular}{|c|c|c|c|c|c|c|}
\hline \multirow[b]{2}{*}{ Type of Utilization } & \multirow[b]{2}{*}{ Unadjusted } & \multicolumn{2}{|c|}{$\begin{array}{l}\text { 95\% Confidence } \\
\text { Interval }\end{array}$} & \multirow[b]{2}{*}{ Adjusted } & \multicolumn{2}{|c|}{$\begin{array}{l}\text { 95\% Confidence } \\
\text { Interval }\end{array}$} \\
\hline & & Lower & Upper & & Lower & Upper \\
\hline & $\begin{array}{l}\text { Observations: } \\
\qquad(\mathrm{n}=304,422) \\
(\text { except ER, } \\
\mathrm{n}=238,340)\end{array}$ & & & $\begin{array}{l}\text { Patients: } \\
\quad(\mathrm{n}=66,638) \\
\quad(\text { except ER, } \\
\mathrm{n}=65,290)\end{array}$ & & \\
\hline \multicolumn{7}{|l|}{ Ambulatory care encounters } \\
\hline Total Mental Health & $-1.9 \%$ & $-2.0 \%$ & $-1.8 \% * * *$ & $0.5 \%$ & $0.2 \%$ & $0.9 \%$ *** \\
\hline Mental Health Specialty & $-1.2 \%$ & $-1.4 \%$ & $-1.1 \% * * *$ & $-1.0 \%$ & $-1.6 \%$ & $-0.3 \% * * *$ \\
\hline Primary Care & $-0.3 \%$ & $-0.3 \%$ & $-0.2 \% * * *$ & $-0.2 \%$ & $-0.7 \%$ & $0.3 \%$ \\
\hline Specialty & $0.0 \%$ & $-0.1 \%$ & $0.1 \%$ & $-0.02 \%$ & $-0.3 \%$ & $0.2 \%$ \\
\hline \multicolumn{7}{|l|}{ Acute Care visits/stays } \\
\hline VA ED & $-0.7 \%$ & $-0.8 \%$ & $-0.5 \%$ *** & $0.5 \%$ & $-0.1 \%$ & $1.0 \%$ * \\
\hline VA Hospitalizations & $-0.1 \%$ & $-0.3 \%$ & $0.1 \%$ & $0.1 \%$ & $-0.4 \%$ & $0.6 \%$ \\
\hline Costs & $\beta$ & SE & & $\boldsymbol{\beta}$ & SE & \\
\hline Log of VA health care costs $\dagger$ & -0.7 & 0.03 & $* * *$ & -0.2 & 0.2 & \\
\hline
\end{tabular}

${ }^{* * *} \mathrm{p}<0.01,{ }^{* *} \mathrm{p}<0.05,{ }^{*} \mathrm{p}<0.1$.

ED, emergency department; ER, emergency room; VA, Veterans Affairs.

We report incidence rate ratios (and confidence intervals) from multilevel negative binomial regression models predicting healthcare utilization and coefficients (and standard errors) from multilevel linear model predicting log-transformed total VA costs. Models contained fixed effects for year and clinic and random effects for patient. We adjusted each model for PACT implementation support (Evidence-Based Quality Improvement in Patient Aligned Care Team [EBQI-PACT] status), and patient characteristics (age, gender, race/ethnicity, marital status, VA eligibility, disability service connection, health insurance, homelessness, distance from home to primary care clinic, Charlson Comorbidity Index, mental health diagnoses) for the 5-year study period.

Results for several covariates in adjusted Total Mental Health model: PACT implementation support (EBQI-PACT) (difference $=-0.02 \%$;
$\mathrm{CI}=-0.3 \%, 0.2 \% ; P=.02) ;$ age $($ difference $=$ $-0.01 \% ; \mathrm{CI}=-0.02 \%,-0.01 \% ; P<.001)$; gender $($ difference $=-0.2 \%$; CI $=-0.2 \%$, $-0.1 \% ; P<.001)$; single (difference $=0.1 \%$; $\mathrm{CI}=0.1 \%, 0.2 \% ; P<.001)$; having a serviceconnected disability (difference $=0.3 \%$; $\mathrm{CI}=$ $0.2 \%, 0.4 \% ; P<.001)$; uninsured (difference $=$ $0.1 \% ; \mathrm{CI}=0.02 \%, 0.1 \% ; P=.01)$; homeless $($ difference $=1.2 \% ; \mathrm{CI}=1.1 \%, 1.3 \% ; P<.001)$; distance to clinic (difference $=0.004 \%$; CI $=$ $-0.01 \%, 0.002 \%)$; multiple chronic comorbidities $($ difference $=0.1 \% ; \mathrm{CI}=0.05 \%, 0.1 \% ; P=$ .001 ); having any mental health diagnosis (e.g., depression difference $=1.1 \% ; \mathrm{CI}=1.1 \%, 1.2 \%$; $P<.001 ;$ schizophrenia difference $=0.8 \% ; \mathrm{CI}=$ $0.7 \%, 1.0 \% ; P<.001$ ), except sociopathy (difference $=-0.2 \% ; \mathrm{CI}=-0.3 \%,-0.05 \% ; P=$ $.01)$. 